\title{
Complications after long-term inferior vena cava filter placement
}

\author{
Mary J. Tao MD, Graham Roche-Nagle MD MBA
}

— Cite as: CMAJ 2017 July 4;189:E892. doi: 10.1503/cmaj.161031

A

41-year-old man presented to the emergency department with back pain and bilateral peripheral edema. His medical history included antiphospholipid antibody syndrome. Seven years earlier, he had a massive pulmonary embolism that required embolectomy and placement of a preoperative temporary inferior vena cava filter for prevention of pulmonary embolism postoperatively when anticoagulation treatment was contraindicated. The filter was not removed after the patient's recovery from surgery.

Computed tomography (CT) showed no opacification of the inferior vena cava at the filter apex down to the common iliac and femoral veins bilaterally, indicative of extensive thrombosis occlusion (Figure 1). Additional findings included a posterior tilt of the filter with strut penetration through the inferior vena cava into the surrounding retroperitoneum (Appendix 1, available at www.cmaj. ca/lookup/suppl/doi:10.1503/cmaj.161031/-/DC1).

Given the clot burden and complete occlusion of the inferior vena cava, we left the temporary filter in situ, without additional intervention. Subsequently, the patient was placed on long-term treatment with apixaban because of poorly controlled anticoagulation and rivaroxaban intolerance. He has regular follow-up visits at the thrombosis clinic.

Retrievable filters for the inferior vena cava have become increasingly popular as a treatment modality for venous thromboembolism. The only widely accepted indications for this filter are in patients with acute venous thromboembolism and absolute contraindications to anticoagulation or in those who have failed adequate anticoagulation. ${ }^{1}$

A recent systematic review of clinical trials evaluating retrievable filters and reported complications to the manufacturer and the US Food and Drug Administration found a retrieval rate of $34 \%$ for retrievable filters. ${ }^{2}$ Nonretrieved devices pose a major health concern because longer indwelling times are associated with higher risk of potential complications, including caval perforation or thrombosis, filter fracture and fragment embolization, cardiac perforation and death. ${ }^{3}$

In 2016, Health Canada issued a safety alert that recommended careful consideration of the indications for filter placement and encouraged institutions to develop follow-up programs to ensure prompt removal of filters in patients without ongoing contraindications to anticoagulation. ${ }^{4}$ Some evidence suggests that the risk and benefit profile begins to favour device removal between 29 and 54 days postinsertion if the transient risk of pulmonary embolism subsides. ${ }^{1}$

\section{References}

1. Morales JP, Li X, Irony TZ, et al. Decision analysis of retrievable inferior vena cava filters in patients without pulmonary embolism. J Vasc Surg Venous Lymphat Disord 2013;1:376-84.

2. Angel LF, Tapson V, Galgon RE, et al. Systematic review of the use of retrievable inferior vena cava filters. J Vasc Interv Radiol 2011;22:1522-1530.e3.

3. Joels CS, Sing RF, Heniford BT. Complications of inferior vena cava filters. Am Surg 2003;69:654-9.

4. Inferior vena cava (IVC) filters - Risk of serious complications. Ottawa: Health Canada; 2016. Available: http://healthycanadians.gc.ca/recall-alert-rappel-avis/ hc-sc/2016/59518a-eng.php\#issue-problem (accessed 2016 July 28).

\section{Competing interests: None declared.}

This article has been peer reviewed.

The authors have obtained patient consent.

Affiliations: Division of Vascular Surgery (Tao, Roche-Nagle), Peter Munk Cardiac Centre, Toronto General Hospital, University Health Network; Faculty of Medicine (Tao), University of Toronto, Toronto, Ont.

Correspondence to: Graham Roche-Nagle, Graham.Roche-Nagle@ uhn.ca 